Psychological Medicine, 1983, 13, 469-477

Printed in Great Britain

\title{
EDITORIAL
}

\section{Relapse in schizophrenia: a review of the concept and its definitions ${ }^{1}$}

The increasingly rigorous attention paid to the diagnosis of schizophrenia has not yet been extended to the development of reliable and valid criteria for describing the course of the illness. Over the past two decades the concept of 'relapse' has become the pre-eminent measure of the success of community treatment programmes of schizophrenia. However, to date there has been little effort to examine this measure critically, or to develop other measures to reflect the wide range of clinical outcome in schizophrenia. This issue is not new. Kraepelin has been faulted for his lack of clarity in discussing the association between the diagnosis of dementia praecox and a poor clinical outcome (WHO, 1975). Stressing that 'the general course of dementia praecox is very variable', he outlined several typical courses of the illness, all of which proceeded to terminal dementia (Kraepelin, 1919). He recognized the lack of specifically defined outcome criteria, citing reports showing recovery rates varying from 0 to $25 \%$.

Although Kraepelin remained sceptical that full recovery without any residual defect was possible, Meyer (1922) contended that functional recovery was possible and proposed that, in many cases, schizophrenia could be regarded as episodic, with relapses representing fresh attacks of the disease. Kraepelin acknowledged that 'more than half exhibit marked improvement', but noted that these recoveries 'give way sooner or later to a relapse'. In support of his thesis of incomplete remission, he observed that subsequent relapses almost always consisted of symptoms similar to those presented in the original episode. This consistency of symptom patterns was also reported by Bleuler (1911), who supported the notion that complete restoration of premorbid mental health was a rarity. Bleuler cautioned against attributing much significance to published reports regarding 'cure' rates, not only because of the subjective nature of such determinations, but also because of 'the varying conditions of admission and relapse for each institution', which often play a greater role in defining discharge status than objective changes in psychopathology.

Concern about the natural course of schizophrenia led these pioneers to focus discussion more upon the definition of remission, than its counterpart, relapse. Although concern regarding the course of the illness has continued (Vaillant, 1964; Huber et al. 1981), little attention has been paid to this matter in studies investigating the effectiveness of clinical interventions. With the advent of newer pharmacological and psychosocial interventions, emphasis has shifted to factors surrounding clinical exacerbation after the patient has been discharged from the hospital in a state of partial or complete clinical remission. The effectiveness of community aftercare has been measured in terms of failure to sustain these 'remissions' and, more often than not, by readmission of the patient to hospital. The latter is primarily a social intervention which, although usually associated with symptom exacerbation, is often more closely related to behavioural disturbance - specifically, impending suicidal or homocidal behaviour, or an inability to function in a socially acceptable manner (Wing, 1968). Although there is often an association between the severity of symptoms and behavioural disturbance, the relationship cannot be assumed to be unswerving. Violent behaviour, suicide attempts, and noisy disruptive behaviour are less well tolerated than withdrawn, stuporous behaviour. Persons exhibiting the former kinds of disturbance are more likely to be actively removed from their community habitats than the latter, even though the underlying psychopathology may be of similar severity.

With the increasing emphasis on community management of schizophrenia, patients demonstrating severe, persistent, florid symptoms are more frequently cared for at home. Because such symptoms

1 Address for correspondence: Dr I. R. H. Falloon, U.S.C. Department of Psychiatry, 1934 Hospital Place, Los Angeles, CA 90033, USA. 
are not readily assessed in terms of relapse/remission criteria, these patients are often excluded from research studies (Leff, 1973). Yet they are among those most in need of investigation with new, potentially efficacious, treatment methods.

\section{A TEN-YEAR REVIEW OF THE RELAPSE CONCEPT}

This paper will examine the use of 'relapse' as a major outcome variable in controlled community outcome studies of schizophrenia. Major psychiatric journals ${ }^{1}$ which publish clinical research studies have been surveyed for the last 10 years. From 1971 to 1980 , there are 18 articles (published or referenced) which report findings from randomized, controlled, outcome studies of schizophrenic out-patients of at least 6 months duration.

In undertaking this endeavour, the authors sought to answer the following questions: (1) How have investigators operationally defined 'relapse' when presenting outcome data? (2) Are the definitions comparable? (3) Has attention been paid to methodological issues such as the need for independent, treatment-blind raters, and the need to demonstrate an acceptable degree of inter-rater reliability in making this assessment? (4) Have alternative methods of assessing the severity of schizophrenia been effectively employed? A summary of this review is provided in the Appendix.

\section{Definitions of relapse}

Despite the widespread use of 'relapse' in clinical settings, only 9 of the $18(50 \%)$ controlled outcome studies explicitly employed this term to delineate a deterioration of clinical state. Six other studies $(33 \%)$ used the relapse concept in the determination of the end-point of treatment-related withdrawals from the follow-up studies. The 3 remaining studies measured the severity of psychopathology without employing the relapse concept.

A detailed examination of the definitions of 'relapse' and comparable criteria (i.e. 'treatment withdrawal', 'treatment failure', 'acute exacerbation', 'deterioration in clinical condition') revealed a wide variation of the criteria employed. These included: hospital readmission, exacerbation of all symptoms, exacerbation of symptoms of schizophrenia, and the need for a major change in clinical management.

\section{(a) Hospital readmission}

This was employed as a major determinant of relapse in $6(40 \%)$ of the 15 studies that employed the relapse concept (Abuzzahab \& Zimmerman, 1980; del Guidice et al. 1975; Hogarty et al. 1973, 1979; Linn et al. 1979; Schooler et al. 1980). The two studies of Hogarty and his colleagues incorporated the notion of impending hospital readmission and actual hospital readmission in their definition of relapse. None of the studies specifically linked readmission to hospital to specific exacerbations of schizophrenia, although most claimed that exacerbations in clinical state (unspecified) were associated with the admissions. Details provided in the methods and results sections of several studies indicated that these exacerbations included affective episodes, suicide attempts and toxic episodes, as well as increased severity of specific symptoms of schizophrenia (Hogarty et al. 1979; Schooler et al. 1980).

The use of hospital readmission as an indicator of failure of community treatment has merit in being readily operationalised, especially where there is a strong conviction that hospital admission is to be avoided at all costs. Undoubtedly, a major proportion of hospital readmissions of patients who have suffered a clearly defined schizophrenic illness are contingent on a reappearance or worsening of the symptoms of schizophrenia. However, this is by no means the sole reason for hospital care. Falloon et al. (1978) observed that hospital admission was associated with affective episodes as often as it was linked with schizophrenic exacerbations. The pathogenesis of depression in schizophrenia is unclear (Hirsch, 1982; McGlashan \& Carpenter, 1976; Sheldrick et al. 1977).

\footnotetext{
1 These journals included: Acta Psychiatrica Scandinavica, American Journal of Psychiatry, Archives of General Psychiatry, British Journal of Psychiatry, British Medical Journal, Comprehensive Psychiatry, Journal of Nervous and Mental Disease, Psychological Medicine, Psychopharmacology Bulletin, and Schizophrenia Bulletin.
} 
However, at this stage of our knowledge, episodes of affective disorders which occur in the absence of florid schizophrenic symptoms are probably best measured separately.

Hospital readmission is a significant event in the course of schizophrenia that is indicative of a chronic relapsing course. Nevertheless, in present day psychiatry, where crisis treatment and rapid return to the community are advocated, hospital stay may be very brief and represents a relatively small aberration during the course of the illness. Only one study (Linn et al. 1979) examined the duration of hospital stay. One comparative drug study that did not employ the relapse concept excluded entirely the small number of hospital admissions from the data analysis (Chouinard $e t$ al. 1978).

While such variation in the choice of relapse criteria may seem arbitrary, it is salutary to note that it may lead to substantial variation in the reported 'relapse rates'. For example, if Leff \& Wing (1971) had employed hospital admission as a major criterion for 'relapse' in their drug-placebo comparison study, the significant difference favouring the active drug group would have been obscured. These investigators reported that only half the placebo 'relapses' required readmission compared with all the active drug 'relapses'. A sceptical interpretation of these data might suggest that the placebo 'relapses', while more frequent, were less severe thain the drug 'relapses' - an important distinction for the clinician. The same investigators have pointed out in other publications (Wing, 1968; Vaughn \& Leff, 1976) that the decision to admit a patient to hospital is frequently determined by the tolerance of community support systems, especially the family, and is not based exclusively on the severity of illness. This socially mediated effect has not been considered in the presentation of rehospitalization data. Although randomization provides some control in drug comparisons, with the trend towards examining psychosocial interventions which aim to enhance social support and tolerance, a reduction in hospital admission might be erroneously equated with a reduction in the severity of symptoms.

Admission to hospital remains an important issue in community management and, for this reason, should retain an important position in comparative outcome data. The serious limitations described above detract from the pre-eminence accorded to it in the outcome literature of the past decade.

(b) Clinical deterioration: non-specific

Nine of the studies $(50 \%)$ have employed unspecified 'clinical deterioration', with or without hospital admission, as a major criterion for their definition of relapse. Few studies provide details of the major features of such deterioration but imply that these exacerbations are florid episodes of schizophrenia. Those studies which do provide descriptions of the episodes of deterioration suggest that such assumptions may be unwarranted. Suicide attempts in the absence of florid symptoms of schizophrenia have been considered 'relapses' in some studies (Rifkin et al. 1977; Quitkin et al. 1978; Hogarty et al. 1979). Affective episodes, both depressive and hypomanic, as well as situational reactions and toxic drug effects were among the episodes included in the broad definition of relapse employed in several data analyses. It may be argued that the considerable overlap between affective and schizophrenic phenomena in many patients presents difficulty in discriminating the dominant psychopathology of exacerbations, and that the general clinical management is quite similar. Such clinical pragmatism has little place in controlled outcome research that aims not merely to determine the relative merits of two or more therapeutic interventions but also to increase understanding of the psychopathology of mental illness. To answer questions concerning overall morbidity, schizophrenic and affective episodes may be combined but, in addition, separate analyses are recommended. Crucial questions such as whether neuroleptic drugs, especially intramuscular fluphenazine, may contribute to an excess of depression while controlling schizophrenia can only be resolved in this manner (Hirsch et al. 1973; Falloon et al. 1978; Schooler et al. 1980).

(c) Clinical deterioration: specific

Only a third (5/15) of the studies employing relapse defined this in terms of clinical deterioration of specific florid schizophrenic phenomenology (Falloon et al. 1978; Hirsch et al. 1973; Kane et al. 
1979; Leff \& Wing, 1971; Rifkin et al. 1977). The definition in one study employed the term 'psychotic' rather than 'schizophrenic', but this appeared to be a purely semantic distinction (Kane et al. 1979). It is possible that other studies restricted their assessments of relapse to schizophrenic features but, in the absence of detailed descriptions of their criteria, it is not wise to speculate.

\section{(d) Change of management requiring termination}

Early termination of subjects in placebo-controlled outcome studies when significant deterioration is observed is an essential ethical procedure. Unfortunately, the decision to terminate may be a response to a wide range of clinical outcomes. Eight studies $(53 \%)$ incorporated this criterion into the definition of relapse. One drug study provided specific dosage adjustment limits that, when exceeded as a result of clinical deterioration, necessitated termination (Schooler et al. 1980). This operational specificity was absent in the other studies which cited the use of management change as a relapse criterion. In one study the signs of 'imminent' relapse were sufficient criteria for termination (Kane et al. 1979). In another, the criterion used was the clinician's 'concern' about the patient's clinical state requiring confirmation that he/she was receiving an active drug (Leff \& Wing, 1971). Such a definition suggests that, in placebo studies, the thresholds for termination are determined largely by 'therapist apprehension' and may correlate less well with the severity of schizophrenia. Recent reports by Herz and his colleagues indicate that many of the prodromal symptoms of impending relapse are non-specific tension and somatic disturbance that may equally well portend an affective disturbance as an exacerbation of schizophrenia (Herz \& Melville, 1980). No study reported the subsequent development of definite schizophrenic exacerbations in the early termination group.

\section{(e) Social impairment}

Levels of social role functioning are important considerations in the evaluation of outcome of therapies for schizophrenia. Deterioration in work and interpersonal roles are commonly associated with an increase in florid schizophrenic symptoms. In 3 studies reviewed the operational definition of relapse explicitly included 'marked' or 'significant' social impairment as a major criterion (Hogarty et al. 1979; Quitkin et al. 1978; Rifkin et al. 1977). As discussed earlier, the use of rehospitalization criteria by other investigators could be considered an indirect measure of social impairment. This confusion between exacerbation of symptoms and the subsequent level of social morbidity is evident. Premorbid adjustment, coping ability and baseline levels of social function are all potential determinants of social impairment. The dilemma is illustrated in the following passage from Rifkin et al. (1977, p. 44).

Relapse was not defined using strict operational criteria, but rather took into account the patient's base line state and particular circumstances. For example, an asymptomatic college student would be terminated sooner, on the development of cognitive confusion, than someone who was not working and continuing to have considerable interpersonal difficulties, although he was in remission.

Such multi-determined criteria for relapse are difficult to support in terms of scientific rigour, yet such candid descriptions of the methods employed provide an excellent illustration of the inadequacies of the deployment of the clinical relapse concept as a specific measure of the severity of schizophrenia. Exacerbation of the symptoms of schizophrenia, while usually associated with social morbidity, may occur without substantial changes in social functioning and should be measured independently.

Other studies have employed independent assessment of social functioning at relapse and have restricted the definition of relapse to clinical outcome (Abuzzahab \& Zimmerman, 1980; Clark et al. 1975; Falloon et al. 1978; Hirsch et al. 1973; Hogarty et al. 1973; Linn et al. 1979; O'Brien et al. 1972; Schooler et al. 1980). 


\section{Psychometric aspects of relapse assessment}

A review of the assessment procedures employed in defining relapse revealed that, in all instances, the major determinant relied on the clinical judgement of the assessor without using objective rating scales. Although almost all studies incorporated standardized assessment instruments in addition to clinical judgement, none used the contribution of these rating scales to the determination of relapse. In most studies the rating scales were administered after it was agreed that a relapse had occurred. In those studies which employed admission to hospital as a major criterion for relapse, the precise nature of that decision-making process was not clearly specified. While several studies employed a consensus between two or more assessors in defining relapse, none reported the levels of inter-rater concordance.

Two-thirds of the studies employed double-blind methodology, but relapse was usually determined by persons who were closely involved in the treatment process, most commonly the prescribing psychiatrist or clinical team. Hirsch and his colleagues (1973) undertook a comparison between the clinical determination of relapse and several other sources of information, including deterioration on the Present State Examination (PSE) and interviews with a close informant. No deterioration on the PSE was assessed in $26 \%$ of clinical relapses, while an informant's observations of increased symptoms disagreed with relapse determination in only $15 \%$ of the cases. These data support the need for a closer scrutiny of relapse assessment procedures and suggest substantial error variance that has not been accounted for in the interpretation of the results. The independence, blindness to treatment, and reliability of assessment are important methodological concerns that appear deficient in this body of work.

\section{Studies that did not use 'relapse'}

Three studies $(17 \%)$ did not use any relapse concepts in the longitudinal assessment of the course of schizophrenia (Chouinard et al. 1978; Claghorn et al. 1974; Donlon \& Meyer, 1978). Instead, they examined rating scales of severity of psychopathology at several points.

The assessment of relapse concerns signficant changes in severity at any time during the prescribed follow-up period, at which time the patient is removed from the study and his subsequent course is uncharted. Serial assessment on standardized rating scales provides a longitudinal measure of the course of the illness that encompasses the potential for improvement as well as exacerbation. The study of Linn and her colleagues (1979) is notable for combining relapse data with serial assessment of psychopathology of each patient over an entire 2-year period. Schizophrenia is usually characterized by remissions and exacerbations, and studies should be designed to focus on the longitudinal course as well as the acute crises.

\section{DISCUSSION}

It is apparent that the use of relapse as the major criterion for assessing community outcome of schizophrenia suffers from serious deficiencies. Operational definitions in published reports lack precise descriptions of assessment procedures and suggest that, at best, the determination is an expedient clinical judgement of functional deterioration of a patient's clinical, behavioural and social status. A change of clinical management such as admission to hospital, increase or change of medication, or crisis intervention is generally contingent upon a relapse. Although in most instances an increase in the severity of the schizophrenic illness accompanies this management crisis, the data presented suggest that there is a less than perfect correlation, and that affective episodes may be categorized as 'relapses' in some studies and not in others. Thus, comparability of 'relapse rates' between studies is not valid. Methodological inadequacies in the assessment of relapse are present in almost all studies, with little attention being paid to the reliability and validity of the measures employed.

The studies reviewed established substantial advantages of neuroleptic drugs over placebo. It is unlikely that the deficiencies noted would significantly affect these well-replicated findings. However, 
in studies which compare the differences between drugs and psychosocial interventions, small but meaningful advantages may be obscured by such imprecise measurement. There are many questions of interest in the community treatment of schizophrenia. These may include: 'Does treatment $(a)$ prevent onset of future episodes (relapse) in remitted patients; $(b)$ reduce the severity of future episodes; $(c)$ prevent exacerbation among non-remitted patients; $(d)$ reduce the severity of persistent symptoms; $(e)$ reduce hospital admission and length of stay; $(f)$ reduce handicap and enhance social functioning; and/or ( $g$ ) prevent affective (and other) episodes?' Only the first of these questions can be effectively answered by the measurement of relapse and that necessitates first defining 'remission' a definition provided in only one of the studies reviewed (Kane et al. 1979). Definitions of relapse have encompassed various combinations of the seven outcome variables listed above and very few studies have presented these measures separately. A combination of criterion measures of relapse, remission, hospital admission and length of stay with rating scale measures of severity of psychopathology (including 'negative' and florid schizophrenic symptoms, as well as other psychiatric phenomenology) and social functioning is advocated for the comprehensive evaluation of the community outcome of schizophrenia.

Recent studies have shown an increasing tendency to utilize 'survival' time prior to readmission or relapse as a measure of community tenure (Abuzzahab \& Zimmerman, 1980; del Guidice et al. 1975; Falloon et al. 1978; Hogarty et al. 1979; Linn et al. 1979; Schooler et al. 1980). This enables investigators to discriminate between treatments that delay, but do not necessarily prevent, relapse, and represents an advance in the utility of the relapse concept. The analogy with a terminal illness is unfortunate. It equates relapse with death and thereby casts a pall over community rehabilitation efforts. Current effective treatment of exacerbations of schizophrenia provides rapid restabilization which often can be achieved on an out-patient basis or with a brief hospital admission.

The measurement of clinical severity of schizophrenia is not an easy task. For this the re-emergence of readily recognized florid symptoms in a previously symptom-free person holds clear advantages. However, despite the improved overall prognosis noted in recent years, perhaps $50 \%$ of all patients suffering from schizophrenia do not attain a stable clinical remission (Bleuler, 1974; Ciompi, 1980). Studies that restrict selection solely to remitted patients limit their generalization to the entire population with schizophrenia (Leff, 1973). Furthermore, reappearance of florid symptoms may be brief and mild, bearing little resemblance to a major episode.

The use of serial rating scale assessments may assist in quantifying the severity of such episodes. A further development has been the use of serial ratings of 'target' symptoms. This approach has been effectively employed with neurotic disorders (Gelder et al. 1967; Sloane et al. 1975). It entails the definition, on a case by case basis, of the specific schizophrenic symptoms that each patient is known to have exhibited prominently during prior exacerbations, and the rating of their severity at regular intervals, and during suspected exacerbations. Care is taken to select unambiguous schizophrenic symptoms that are unlikely to overlap with affective episodes or toxic drug-effects. Thus a 'delusion of external control' would be preferred to 'social withdrawal'. Severity can be measured on linear severity scales similar to other psychopathology measures such as the Psychiatric Assessment Scale (Krawiecka et al. 1977) or the Brief Psychiatric Rating Scale (Overall \& Gorham, 1962). Specific rating scales of severity of schizophrenia are yet to be developed, although current attempts are in progress (Chapman \& Chapman, 1980).

The emphasis on relapse and exacerbation has drawn attention to relatively infrequent, often brief, management crises and away from the long-term course of schizophrenia. The absence of florid exacerbations cannot be equated with the absence of illness. The term 'negative' symptom has been coined to describe the less dramatic, yet potentially disabling features of flattened affect, social withdrawal, lack of motivation and poverty of speech that are associated with schizophrenia (Crow, 1980). Bleuler (1911) considered these to be the primary features of schizophrenia, upon which the more labile, secondary symptoms of delusions and hallucinations are superimposed at times of stress.

Successful management of an illness should effectively minimize all symptoms of the condition and any associated disability and handicap. At this point, clinical ratings of relapse appear to dominate research in the community management of schizophrenia. To ensure further advance it 
is crucial that these crude measures are refined so that high standards of scientific endeavour can be maintained in this extremely important area of psychiatry.

IAN R. H. FALLOON, GRANT N. MARSHALL, JEFFREY L. BOYD, JAVAD RAZANI AND CATHY WOOD-SIVERIO

\section{REFERENCES}

Abuzzahab, F. S. \& Zimmerman, R. L. (1980). Factors determining patient tenure on a 3-year double-blind investigation of pimozide versus fluphenazine $\mathrm{HCl}$. Advances in Biochemical Psychopharmacology 24, 547-550.

Bleuler, E. (1911). Dementia Praecox: Or the Group of Schizophrenias (transl. J. Zinkin). International Universities Press: New York.

Bleuler, M. (1974). The long-term course of the schizophrenic psychoses. Psychological Medicine 4, 244-254.

Chapman, L. J. \& Chapman, J. P. (1980). Scales for rating psychotic and psychotic-like experiences as continua. Schizophrenia Bulletin 6, 476-489.

Chouinard, G., Annable, L. \& Kropsky, M. (1978). A double-blind controlled study of pipothiazine palmitate in the maintenance treatment of schizophrenic outpatients. Journal of Clinical Pharmacology 18, 148-154.

Ciompi, L. (1980). Catamnestic long-term study on the course of life and aging of schizophrenics. Schizophrenia Bulletin 61, 606-618.

Claghorn, J. L., Johnstone, E. E. \& Cook, T. H. (1974). Group therapy and maintenance treatment of schizophrenics. Archives of General Psychiatry 31, 361-365.

Clark, M.L., Huber, W. K. \& Hill, D. (1975). Pimozide in chronic schizophrenic outpatients. Diseases of the Nervous System 36, 137-141.

Crawford, R. \& Forrest, A. (1974). Controlled trial of depot fluphenazine in outpatient schizophrenics. British Journal of Psychiatry 124, 385-391.

Crow, T. J. (1980). Molecular pathology of schizophrenia: more than one disease process? British Medical Journal 280, 66-68.

del Guidice, J., Clark, W. G. \& Gocka, E. F. (1975). Prevention of recidivism of schizophrenics treated with fluphenazine enanthate. Psychosomatics 16, 32-36.

Donlon, P. T. \& Meyer, J. E. (1978). A twelve month comparison of penfluridol and trifluoperazine in chronic schizophrenic outpatients. Journal of Clinical Psychiatry 39, 582-587.

Falloon, I. R. H., Watt, D. C. \& Shepherd, M. (1978). A comparative controlled trial of pimozide and fluphenazine decanoate in the continuation therapy of schizophrenia. Psychological Medicine 8, 59-70.

Gelder, M. G., Marks, I. M. \& Wolff, H. H. (1967). Desensitization and psychotherapy in the treatment of phobic states: A controlled inquiry. British Journal of Psychiatry 113, 53-73.

Herz, M. I. \& Melville, C. (1980). Relapse in schizophrenia. American Journal of Psychiatry 137, 801-805.

Hirsch, S. R. (1982). Depression revealed in schizophrenia. British Journal of Psychiatry 140, 421-422.

Hirsch, S. R., Gaind, R. \& Rohde, P. D. (1973). Outpatient management of chronic schizophrenic patients with long-acting fluphenazine: double-blind placebo trial. British Medical Journal i, 633-637.

Hogarty, G. E., Goldberg, S. C. \& NIMH Collaborative Study Group (1973). Drug and sociotherapy in the aftercare of schizophrenic patients: one-year relapse rates. Archives of General Psychiatry 28, 54-64.

Hogarty, G. E., Schooler, N. \& Ulrich, R. (1979). Fluphenazine and social therapy in the aftercare of schizophrenic patients: relapse analyses of a two-year controlled study of fluphenazine decanoate

and fluphenazine hydrochloride. Archives of General Psychiatry 36, 1283-1294.

Huber, G., Cross, G. \& Schuttler, R. (1981). Longitudinal studies of schizophrenic patients. Schizophrenia Bulletin 6, 592-605.

Kane, J. M., Rifkin, A. \& Quitkin, F. (1979). Low dose fluphenazine decanoate in maintenance treatment of schizophrenia. Psychiatry Research 1, 341-348.

Kraepelin, E. (1919). Dementia praecox and paraphrenia. In The Textbook of Psychiatry (transl. R. M. Barclay). Robert E. Krieger: New York.

Krawiecka, M., Goldberg, D. \& Vaughan, M. (1977), A standardized psychiatric assessment scale for rating chronic psychotic patients. Acta Psychiatrica Scandinavica 55, 299-308.

Leff, J. P. (1973). Influence of selection of patients on results of clinical trials. British Medical Journal iv, 156-158.

Leff, J. P. \& Wing, J. K. (1971). Trial of maintenance therapy in schizophrenia. British Medical Journal iii, 559-604.

Linn, M. W., Caffey, E. M. \& Klett, J. (1979). Day treatment and psychotropic drugs in the aftercare of schizophrenic patients. Archives of General Psychiatry 36, 1055-1066.

McGlashan, T. H. \& Carpenter, W. T. Jr (1976). Postpsychotic depression in schizophrenia. Archives of General Psychiatry 33, 231-239.

Meyer, A. (1922). Constructive formulation of schizophrenia. American Journal of Psychiatry 1, 355.

O'Brien, C. P., Hamm, K. B. \& Ray, B. A. (1972). Group vs. individual psychotherapy with schizophrenics. Archives of General Psychiatry 27, 474-478.

Overall, J. E. \& Gorham, D. R. (1962). The Brief Psychiatric Rating Scale. Psychological Reports 10, 799-812.

Quitkin, F., Rifkin, A. \& Kane, J. (1978). Long-acting oral vs. injectable antipsychotic drugs in schizophrenics. Archives of General Psychiatry 35, 889-892.

Rifkin, A., Quitkin, F. \& Rabiner, C. J. (1977). Fluphenazine decanoate, fluphenazine hydrochloride given orally, and placebo in remitted schizophrenics: I. Relapse rates after one year. Archives of General Psychiarry 34, 43-47.

Schooler, N. R., Levine, J. \& Severe, J. B. (1980). Prevention of relapse in schizophrenia: an evaluation of fluphenazine decanoate. Archives of General Psychiatry 37, 16-24.

Sheldrick, C., Jablensky, A., Sartorius, N. \& Shepherd, M. (1977). Schizophrenia succeeded by affective illness: catamnestic study and statistical inquiry. Psychological Medicine 7, 619-624.

Sloane, R. B., Staples, F. R. \& Cristol, A. H. (1975). Psychotherapy versus Behaviour Therapy. Harvard University Press: Cambridge, Mass.

Valliant, G. E. (1964). Prospective prediction of schizophrenic remission. Archives of General Psychiatry 11, 509-518.

Vaughn, C. E. \& Leff, J. P. (1976). The influence of family and social factors on the course of psychiatric illness: a comparison of schizophrenic and depressed neurotic patients. British Journal of Psychiatry 129, 125-137.

Wing, J. K. (1968). Social treatments of mental illness. In Studies of Psychiarry (ed. M. Shepherd and D. L. Davies), pp. 17-31. Oxford University Press: London.

World Health Organization (1975). International Pilot Study of Schizophrenia. WHO: Geneva. 


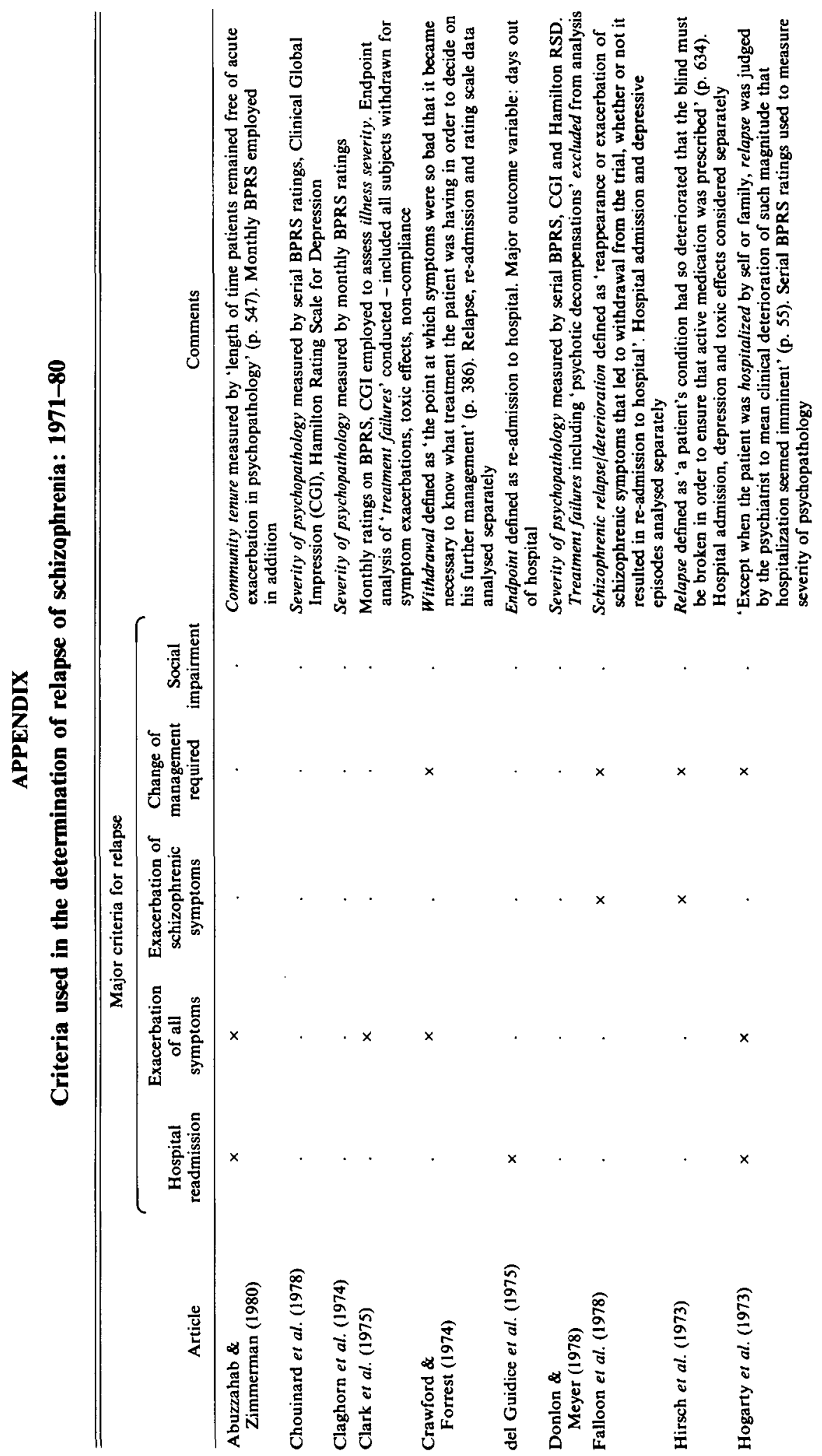




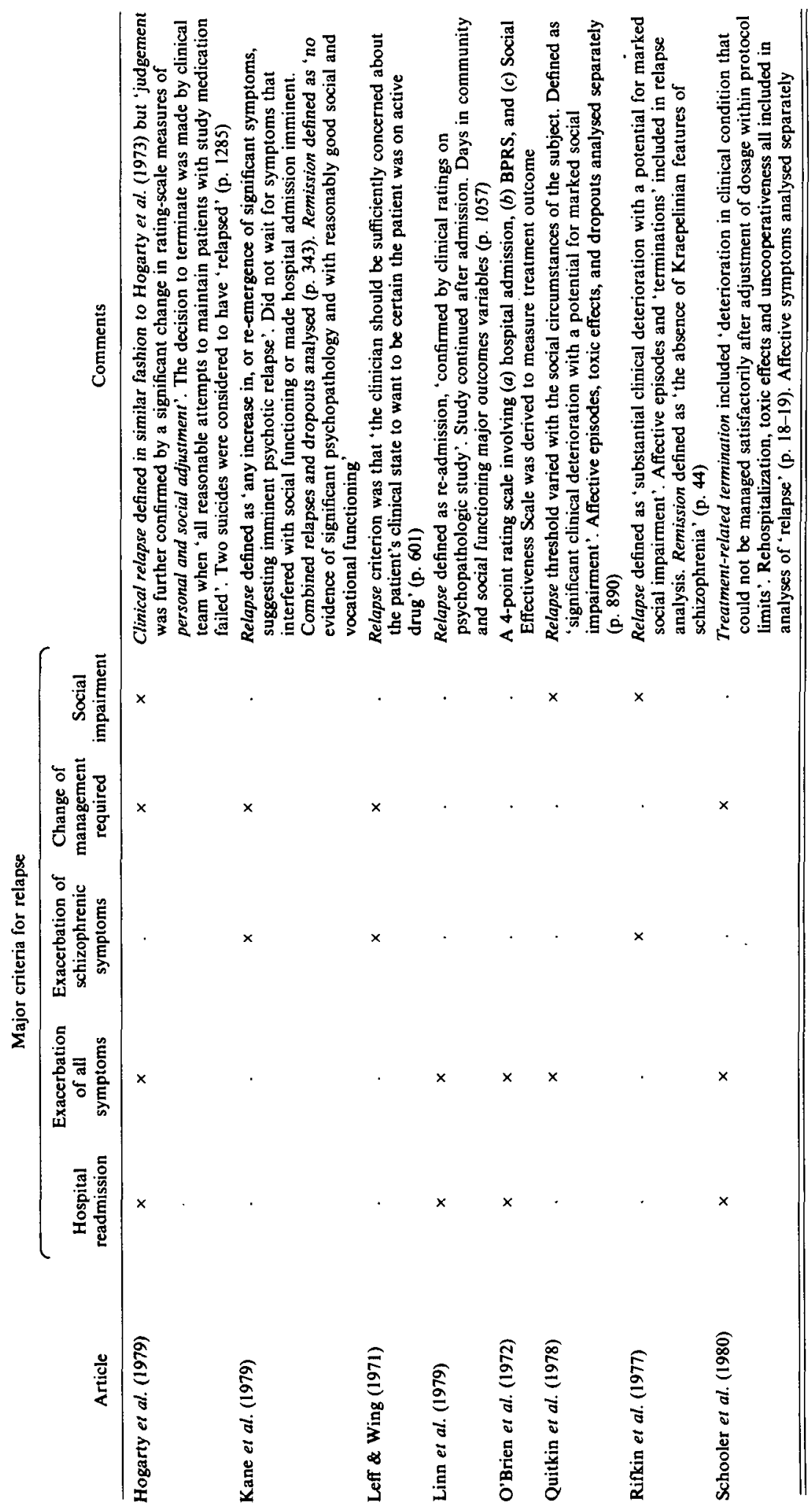

